\title{
The behavior of some finite automata in a non-stationary fuzzy environment
}

\author{
TARIEL KHVEDELIDZE \\ Department of Computer Sciences \\ Tbilisi State University \\ University St. 2, Tbilisi 0143 \\ GEORGIA
}

\begin{abstract}
The behavior of two types of finite automata in a non-stationary fuzzy environment is considered, which, depending on the states of the automata, encourages or punishes them with some fixed membership functions. It is assumed that the behavior of automata in a fuzzy environment is described by generalized ergodic Markov chains and, using the property of such chains, it is shown that the considered automata, under certain conditions on fuzzy punishment functions, are learners and predominantly perform the action for which the sum of fuzzy functions of belonging to punishment is minimal.
\end{abstract}

Key-Words: - behavior of automaton, depth of state, generalized Markov chain, learning automaton, membership function, non-stationary fuzzy environment.

Received: February 28, 2021. Revised: December 2, 2021. Accepted: December 20, 2021. Published: December 31, 2021.

\section{Introduction}

In Markov models of a complex system, finite automata are widely used, both deterministic and probabilistic structures. The idea that finite automata are a very convenient object for constructing mathematical models of complex systems was first expressed by J. von Neumann [1]. However, the direction of work related to the construction of automaton models of behavior was formulated and developed by M. L. Tsetlin. As an elementary behavioral task, he chose the problem of choosing one of several actions with random reinforcement - the task about behavior of an automaton in a random environment. He owns the well-known construction of a finite automaton with linear tactics, which forms an asymptotically optimal sequence in a stationary random environment [2]. Then V.I. Krinsky, according to the rule [3], in which first has been reviewed the problem of sequential selection of one of two ways of action, each of which can lead to success or failure, built a finite-automaton construction and studied its behavior in a stationary random environment [4].

It was believed that the behavior of a finite automaton V. I. Krinsky is asymptotically optimal in any stationary random environment. However, in [5] it is shown that it is not at all this: the behavior of the finite automaton V. I. Krinsky in a stationary random environment can be either optimal or anti-optimal, depending on the initial state of the automaton.
Recently, the theory of fuzzy sets proposed by L. Zadeh [6], has attracted great interest from researchers in various fields. However, if the study of the behavior of the probability systems is based on the classical apparatus of probabilistic Markov chains and has a huge statistical base, then for systems with fuzzy information there is no such statistical base and mathematical apparatus yet. Such an analysis became possible after some generalization of the apparatus of Markov chains to the non-probability case [7]. Using this apparatus, in [8] the behavior of an finite automaton with linear tactics of M. L. Tsetlin's in a stationary fuzzy environment was investigated, and in [9] - the behavior of the finite automaton V.I. Krinsky's, where the formulas are obtained connect fuzzy statements about the internal states of the automaton with its actions.

In this work, based on the finite automata of M. L. Tsetlin's and V.I. Krinsky's, more general algorithm for the behavior of these finite automata and their functioning in a non-stationary fuzzy environment is considered. The fuzzy environment, depending on the states of the automata, encourages or punishes these automata with some fuzzy membership functions. Assuming that the behavior of automata in such an environment is described by generalized ergodic Markov chains and it is shown on the basis of the properties of such chains that the finite automata under consideration, subject to certain conditions on fuzzy punishment functions, are learnable and they 
basically perform an action, for which the sum of fuzzy functions of belonging to the punishment is minimal.

\section{Behavior of a finite automaton $L_{2 n, 2}^{(j)}$ in a non-stationary fuzzy environment}

Consider a finite automaton $L_{2 n, 2}^{(j)}$, which has $2 n$ internal states $L_{2 n}=L_{1}^{(n)} \cup L_{2}^{(n)}$, and $L_{1}^{(n)} \cap L_{2}^{(n)}=\emptyset$. In the states of depth $x=\overline{1, n}$ of the subset $L_{\alpha}^{(n)}$, $\alpha=1,2$ the automaton performs the action $f_{\alpha}$, $\alpha=1,2$. So the state number corresponds to the state depth and the automaton has a finite depth $n$. When encouraged, the automaton from the state of depth $x$ of the subset $L_{\alpha}^{(n)}, \alpha=1,2$ goes into the state of depth $x+1$, at a punishment in the state of depth $x-1, x=\overline{2, n-1}$ in the same subset. The change of actions of the automaton occurs from the state of depth $x=1$ : the automaton, being in the state of depth $x=1$ of the subset $L_{\alpha}^{(n)}, \alpha=1,2$, at a punish, goes in the state of depth $x=j$ in another subset. The graphs of transitions between the states of the finite automaton $L_{2 n, 2}^{(j)}$ are shown in Fig. 1.

In this figure, the upper graph corresponds to the transitions between the states of the automaton at encouragements, and the lower graph - at punishments.

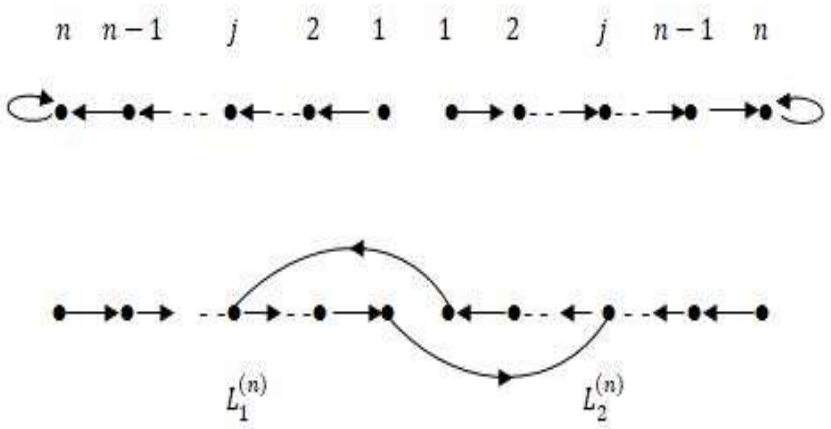

Fig: 1. Graphs of transition between states for a finite automaton $L_{2 n, 2}^{(j)}$ with two actions in the subset

$$
L_{2 n}=L_{1}^{(n)} \cup L_{2}^{(n)}
$$

Note that the automaton $L_{2 n, 2}^{(j)}$ for $j=1$ is a finite linear automaton by M.L. Tsetlin's [3], and for $j=n$ it is a finite automaton with hysteresis tactics [10].
Let the automaton $L_{2 n, 2}^{(j)}$ be placed in a nonstationary fuzzy environment and assume that $\mu_{x}^{(\alpha)}(t)$ means the function of belonging to the states of automaton of depth $x \quad(x=\overline{1, n})$ and corresponding to the action $f_{\alpha}, \alpha=1,2$ at times $t=1,2, \ldots$. . The interaction of an automaton with the environment is determined according to a fuzzy scheme as follows: the automaton being in the state of depth $x$ of the subset $L_{\alpha}^{(n)}, \alpha=1,2$ is punished with the membership function $\lambda_{x}^{(\alpha)}$ or rewarded with the membership function $\left(1-\lambda_{x}^{(\alpha)}\right), \quad x=\overline{1, n}$, $\alpha=1,2$.

We will assume that the system "automaton fuzzy environment" is described by a generalized Markov chain [7], which is ergodic and in it over time "fuzzy flows" are formed $\lambda_{x}^{(\alpha)} \mu_{x}^{(\alpha)}, x=\overline{1, n}$, $\alpha=1,2$.

Then taking into account the properties of the ergodic Markov chain, "fuzzy flows" from $L_{1}^{(n)}$ to $L_{2}^{(n)}$ and back are balance, i.e.

$$
\lambda_{1}^{(1)} \mu_{1}^{(1)}=\lambda_{1}^{(2)} \mu_{1}^{(2)},
$$

and "fuzzy flows" from the state of the automaton in the subset $L_{1}^{(n)}$ of depth $x=k$ in the state of depth $x=k-1$ and back differ in "fuzzy flow" $\lambda_{1}^{(1)} \mu_{1}^{(1)}$ for $k=\overline{2, \jmath}$, and for $k=\overline{j+1, n}$ this difference is compensated by the "fuzzy flow" $\lambda_{1}^{(2)} \mu_{1}^{(2)}$ from the subset $L_{2}^{(n)}$.

Thus,

$$
\begin{gathered}
\lambda_{x}^{(1)} \mu_{x}^{(1)}-\left(1-\lambda_{x-1}^{(1)}\right) \mu_{x-1}^{(1)}=\lambda_{1}^{(1)} \mu_{1}^{(1)}, x=\overline{2, J}, \quad \text { (2) } \\
\lambda_{x}^{(1)} \mu_{x}^{(1)}+\lambda_{1}^{(2)} \mu_{1}^{(2)}-\left(1-\lambda_{x-1}^{(1)}\right) \mu_{x-1}^{(1)}=\lambda_{1}^{(1)} \mu_{1}^{(1)}, \\
x=\overline{J+1, n}
\end{gathered}
$$

and given (1)

$$
\lambda_{x}^{(1)} \mu_{x}^{(1)}-\left(1-\lambda_{x-1}^{(1)}\right) \mu_{x-1}^{(1)}=0, \quad x=\overline{\jmath+1, n},
$$

Similarly, for the subset $L_{2}^{(n)}$ we have:

$$
\begin{aligned}
& \lambda_{x}^{(2)} \mu_{x}^{(2)}-\left(1-\lambda_{x}^{(2)}\right) \mu_{x-1}^{(2)}=\lambda_{1}^{(2)} \mu_{1}^{(2)}, x=\overline{2, J}, \\
& \lambda_{x}^{(2)} \mu_{x}^{(2)}-\left(1-\lambda_{x-1}^{(2)}\right) \mu_{x-1}^{(2)}=0, \quad x=\overline{\jmath+1, n} .
\end{aligned}
$$

Then, from (2) and (3):

$$
\begin{gathered}
\mu_{x}^{(1)}=\frac{\lambda_{1}^{(1)}}{\lambda_{x}^{(1)}}\left\{1+\sum_{i=1}^{x-1} \prod_{l=i}^{x-1} \frac{1-\lambda_{l}^{(1)}}{\lambda_{l}^{(1)}}\right\} \mu_{1}^{(1)}, \\
\mu_{x}^{(1)}=\frac{\lambda_{j}^{(1)}}{\lambda_{x}^{(1)}} \prod_{l=j}^{x-1} \frac{1-\lambda_{l}^{(1)}}{\lambda_{l}^{(1)}} \mu_{j}^{(1)}, \quad x=\overline{\jmath+1, n} .
\end{gathered}
$$

From (4) 


$$
\mu_{j}^{(1)}=\frac{\lambda_{1}^{(1)}}{\lambda_{j}^{(1)}}\left\{1+\sum_{i=1}^{j-1} \prod_{l=i}^{j-1} \frac{1-\lambda_{l}^{(1)}}{\lambda_{l}^{(1)}}\right\} \mu_{1}^{(1)} .
$$

Then, from (5) we will have:

$$
\begin{gathered}
\mu_{x}^{(1)}=\frac{\lambda_{1}^{(1)}}{\lambda_{x}^{(1)}}\left\{1+\sum_{i=1}^{j-1} \prod_{l=i}^{j-1} \frac{1-\lambda_{l}^{(1)}}{\lambda_{l}^{(1)}}\right\} \times \\
\times \prod_{l=j}^{x-1} \frac{1-\lambda_{l}^{(1)}}{\lambda_{l}^{(1)}} \mu_{1}^{(1)}, \quad x=\overline{\jmath+1, n} .
\end{gathered}
$$

Similarly, for the subset $L_{2}^{(n)}$, we have:

$$
\begin{gathered}
\mu_{x}^{(2)}=\frac{\lambda_{1}^{(2)}}{\lambda_{x}^{(2)}}\left\{1+\sum_{i=1}^{x-1} \prod_{l=i}^{x-1} \frac{1-\lambda_{l}^{(2)}}{\lambda_{l}^{(2)}}\right\} \mu_{1}^{(2)}, \\
\mu_{x}^{(2)}=\frac{\lambda_{j}^{(2)}}{\lambda_{x}^{(2)}} \prod_{l=j}^{x-1} \frac{1-\lambda_{l}^{(2)}}{\lambda_{l}^{(2)}} \mu_{j}^{(2)}, \quad x=\overline{J+1, n},
\end{gathered}
$$

From (6) we have:

$$
\begin{gathered}
\mu_{j}^{(2)}=\frac{\lambda_{1}^{(2)}}{\lambda_{j}^{(2)}}\left\{1+\sum_{i=1}^{j-1} \prod_{l=i}^{j-1} \frac{1-\lambda_{l}^{(2)}}{\lambda_{l}^{(2)}}\right\} \mu_{1}^{(2)} . \\
\mu_{x}^{(2)}=\frac{\lambda_{1}^{(2)}}{\lambda_{x}^{(2)}}\left\{1+\sum_{i=1}^{j-1} \prod_{l=i}^{j-1} \frac{1-\lambda_{l}^{(2)}}{\lambda_{l}^{(2)}}\right\} \times \\
\times \prod_{l=j}^{x-1} \frac{1-\lambda_{l}^{(2)}}{\lambda_{l}^{(2)}} \mu_{1}^{(2)}, \quad x=\overline{\jmath+1, n} .
\end{gathered}
$$

Taking into account (1), we express from (6) and (7) all $\mu_{x}^{(2)}, x=\overline{2, n}$ in terms of $\mu_{1}^{(1)}$ :

$$
\begin{array}{r}
\mu_{x}^{(2)}=\frac{\lambda_{1}^{(1)}}{\lambda_{x}^{(2)}}\left\{1+\sum_{i=1}^{x-1} \prod_{l=1}^{i} \frac{1-\lambda_{l}^{(2)}}{\lambda_{l}^{(2)}}\right\} \mu_{1}^{(1)}, \\
\mu_{x}^{(2)}=\frac{\lambda_{1}^{(1)}}{\lambda_{x}^{(2)}}\left\{1+\sum_{i=1}^{j-1} \prod_{l=i}^{j-1} \frac{1-\lambda_{l}^{(2)}}{\lambda_{l}^{(2)}}\right\} \times \\
\times \prod_{l=j}^{x-1} \frac{1-\lambda_{l}^{(2)}}{\lambda_{l}^{(2)}} \mu_{1}^{(1)}, \quad x=\overline{\jmath+1, n} .
\end{array}
$$

Let us now define the fuzzy membership functions $M^{(\alpha)}$ of the state of the automaton to the subset $L_{\alpha}^{(n)}$, in which the automaton performs the action $f_{\alpha}$, $\alpha=1,2$. It is shown in $[8,11]$ that the final membership function $\theta$ coming from $n$ sources $\beta_{1}, \beta_{2}, \ldots, \beta_{n}$ is determined by the expression

$$
=\sum_{i} \beta_{i}-\sum_{i \neq j}^{\theta\left(\beta_{1}, \beta_{2}, \ldots, \beta_{n}\right)=} \beta_{i} \beta_{j}+\sum_{i \neq j \neq k} \beta_{i} \beta_{j} \beta_{k}-\ldots
$$

which can be rewritten in the following form

$$
\begin{gathered}
\theta\left(\beta_{1}, \beta_{2}, \ldots, \beta_{n}\right)= \\
=1-\left(1-\beta_{1}\right)\left(1-\beta_{2}\right) . .\left(1-\beta_{n}\right)= \\
=1-\prod_{i=1}^{n}\left(1-\beta_{i}\right) .
\end{gathered}
$$

Then the final fuzzy membership function $M^{(1)}$ of the state of the automaton of the subset $L_{1}^{(n)}$, in which the automaton $L_{2 n, 2}^{(j)}$ performs the first action,

$$
\begin{gathered}
M^{(1)}=1-\prod_{x=1}^{j}\left(1-\mu_{x}^{(1)}\right) \prod_{x=j+1}^{n}\left(1-\mu_{x}^{(1)}\right)= \\
=1-\left(1-\mu_{1}^{(1)}\right) \times \\
\times \prod_{x=2}^{j}\left(1-\frac{\lambda_{1}^{(1)}}{\lambda_{x}^{(1)}}\left\{1+\sum_{i=1}^{x-1} \prod_{l=i}^{x-1} \frac{1-\lambda_{l}^{(1)}}{\lambda_{l}^{(1)}}\right\} \mu_{1}^{(1)}\right) \times \\
\times \prod_{x=j+1}^{n}\left(1-\frac{\lambda_{1}^{(1)}}{\lambda_{x}^{(1)}}\left\{1+\sum_{i=1}^{j-1} \prod_{l=i}^{j-1} \frac{1-\lambda_{l}^{(1)}}{\lambda_{l}^{(1)}}\right\}\right) \times \\
\times \prod_{l=j}^{x-1} \frac{1-\lambda_{l}^{(1)}}{\lambda_{l}^{(1)}} \mu_{1}^{(1)},
\end{gathered}
$$

and for the final fuzzy membership function $M^{(2)}$ the state of the automaton of the subset $L_{2}^{(n)}$ in which the automaton $L_{2 n, 2}^{(j)}$ performs the second action,

$$
\begin{gathered}
M^{(2)}=1-\prod_{x=1}^{j}\left(1-\mu_{x}^{(2)}\right) \prod_{x=j+1}^{n}\left(1-\mu_{x}^{(2)}\right)= \\
=1-\left(1-\frac{\lambda_{1}^{(1)}}{\lambda_{1}^{(2)}} \mu_{1}^{(1)}\right) \times \\
\times \prod_{x=2}^{j}\left(1-\frac{\lambda_{1}^{(1)}}{\lambda_{x}^{(2)}}\left\{1+\sum_{i=1}^{x-1} \prod_{l=i}^{x-1} \frac{1-\lambda_{l}^{(2)}}{\lambda_{l}^{(2)}}\right\} \mu_{1}^{(1)}\right) \times \\
\times \prod_{x=j+1}^{n}\left(1-\frac{\lambda_{1}^{(1)}}{\lambda_{x}^{(2)}}\left\{1+\sum_{i=1}^{j-1} \prod_{l=i}^{j-1} \frac{1-\lambda_{l}^{(2)}}{\lambda_{l}^{(2)}}\right\}\right) \times \\
\times \prod_{l=j}^{k-1} \frac{1-\lambda_{l}^{(2)}}{\lambda_{l}^{(2)}} \mu_{1}^{(1)},
\end{gathered}
$$

where $\mu_{1}^{(1)}$ is a positive constant such that $\mu_{1}^{(1)} \leq 1$ and its value can be calculated from the obvious equality

$$
M^{(1)}+M^{(2)}=1 .
$$

Let us now analyze the results by comparing the membership function $M^{(1)}$, which is responsible for performing the action $f_{1}$, with the membership function $M^{(2)}$, which is responsible for performing the action $f_{2}$. We will assume that of the two actions 
$f_{1}$ and $f_{2}$, the best is the action for which the sum of fuzzy functions of membership in the punishment is minimal, i.e. if

$$
\sum_{x=1}^{n} \lambda_{x}^{\alpha}<\sum_{x=1}^{n} \lambda_{x}^{\beta}, \quad \alpha, \beta=1,2, \quad \alpha \neq \beta,
$$

then the best action we will consider the action $f_{\alpha}, \alpha=1,2$. To fulfill this inequality, we can consider various possible cases of comparing the parameters $\lambda_{x}^{(\alpha)}$ and $\lambda_{x}^{(\beta)}, x=\overline{1, n}, \alpha, \beta=1,2$, $\alpha \neq \beta$. However, it is easy to verify that it is necessary satisfied if

$$
\begin{gathered}
\max _{x} \lambda_{x}^{(\alpha)}<\min _{x} \lambda_{x}^{(\beta)}, \\
x=1, n, \quad \alpha, \beta=1,2, \quad \alpha \neq \beta .
\end{gathered}
$$

For definiteness, we will assume that

$$
\max _{x} \lambda_{x}^{(1)}<\min _{x} \lambda_{x}^{(2)}, \quad x=\overline{1, n} .
$$

Then it follows from (8) and (9) that $M^{(1)}>$ $M^{(2)}$, that is, the membership function responsible for the action $f_{1}$ will be greater than the membership function responsible for the action $f_{2}$. If at the same time the is also carried inequality

$\max _{x} \lambda_{x}^{(1)}<1 / 2<\min _{x} \lambda_{x}^{(2)}, \quad x=\overline{1, n}$,
then $M^{(1)} \rightarrow 1$ и $M^{(2)} \rightarrow 0$ when $n \rightarrow \infty$.

\section{Behavior of a finite automaton $D_{2 n, 2}^{(j)}$ in a non-stationary fuzzy environment}

Let us now consider the behavior of a finite automaton $D_{2 n, 2}^{(j)}$ in a fuzzy environment. In case of punishment, the transitions between the states of the finite automaton $D_{2 n, 2}^{(j)}$ are the same as those of the finite line automaton $L_{2 n, 2}^{(j)}$, and with encouraged, the automaton $D_{2 n, 2}^{(j)}$ from any state of depth $x$ of the subset $L_{\alpha}^{(n)}, \alpha=1,2$ goes over to the "deepest" state $x=n$ of the same subset. The graphs of transitions between states of the finite automaton $D_{2 n, 2}^{(j)}$ are shown in Fig. 2.

In this figure, the upper graph corresponds to the transitions between the states of the automaton at encouragements, and the lower graph - at punishments.

Note that the automaton $D_{2 n, 2}^{(j)}$ for $j=1$ is a finite automaton of V. I. Krinsky, and for $j=n$ it is a finite automaton of H. Robbins [12].
We assume that the behavior of the automaton $D_{2 n, 2}^{(j)}$ in a fuzzy environment is described by a generalized ergodic Markov chain.

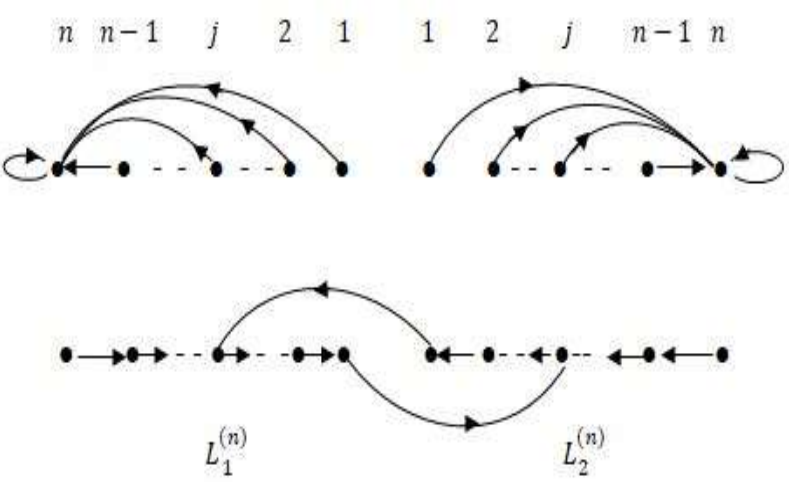

Fig: 2. Graphs of transition between states for a finite automaton $D_{2 n, 2}^{(j)}$ with two actions in the subset

$$
L_{2 n}=L_{1}^{(n)} \cup L_{2}^{(n)}
$$

Then for this automaton, "fuzzy flows" from $L_{1}^{(n)}$ in $L_{2}^{(n)}$ and back is are also balance, i.e. equality is fulfilled

$$
\lambda_{1}^{(1)} \mu_{1}^{(1)}=\lambda_{1}^{(2)} \mu_{1}^{(2)} .
$$

For the balance of "fuzzy flows" between states in the subset $L_{1}^{(n)}$ we have:

$$
\begin{gathered}
\lambda_{x}^{(1)} \mu_{x}^{(1)}-\sum_{i=1}^{x-1}\left(1-\lambda_{i}^{(1)}\right) \mu_{i}^{(1)}=\lambda_{1}^{(1)} \mu_{1}^{(1)}, \\
x=\overline{2, J}, \\
\lambda_{x}^{(1)} \mu_{x}^{(1)}-\sum_{i=1}^{x-1}\left(1-\lambda_{i}^{(1)}\right) \mu_{i}^{(1)}=0, \\
x=\overline{\jmath+1, n} .
\end{gathered}
$$

Similarly, for the subset $L_{2}^{(n)}$, we have:

$$
\begin{gathered}
\lambda_{x}^{(2)} \mu_{x}^{(2)}-\sum_{i=1}^{x-1}\left(1-\lambda_{i}^{(2)}\right) \mu_{i}^{(2)}=\lambda_{1}^{(2)} \mu_{1}^{(2)}, \\
x=\overline{2, j}, \\
\lambda_{x}^{(2)} \mu_{x}^{(2)}-\sum_{i=1}^{x-1}\left(1-\lambda_{i}^{(2)}\right) \mu_{i}^{(2)}=0, \\
x=\overline{\jmath+1, n} .
\end{gathered}
$$

Expressing all $\mu_{x}^{(1)}$ and $\mu_{x}^{(2)}(x=\overline{2, n})$ from (12) $-(15)$ in terms of $\mu_{1}^{(1)}$ and $\mu_{1}^{(2)}$ respectively, we obtain that

$$
\mu_{x}^{(1)}=\lambda_{1}^{(1)}\left(\prod_{i=1}^{x} \lambda_{i}^{(1)}\right)^{-1} \mu_{1}^{(1)}, \quad x=\overline{1, J},
$$




$$
\begin{gathered}
\mu_{x}^{(1)}=\lambda_{1}^{(1)}\left(1-\prod_{i=1}^{j} \lambda_{i}^{(1)}\right)\left(\prod_{i=1}^{x} \lambda_{i}^{(1)}\right)^{-1} \mu_{1}^{(1)}, \\
\mu_{x}^{(2)}=\lambda_{1}^{(2)}\left(\prod_{\substack{i=1 \\
j}}^{x+1, n} \lambda_{i}^{(2)}\right)^{-1} \mu_{1}^{(2)}, x=\overline{1, j}, \\
\mu_{x}^{(2)}=\lambda_{1}^{(2)}\left(1-\prod_{i=1}^{j} \lambda_{i}^{(2)}\right)\left(\prod_{i=1}^{x} \lambda_{i}^{(2)}\right)^{-1} \mu_{1}^{(2)}, \\
x=\frac{j+1, n}{(11),}
\end{gathered}
$$

Taking into account (11), we now express all $\mu_{x}^{(2)}(x=\overline{2, n})$ in terms of $\mu_{1}^{(1)}$. Finally, we will have:

$$
\begin{aligned}
& \mu_{x}^{(2)}=\lambda_{1}^{(1)}\left(\prod_{i=1}^{x} \lambda_{i}^{(2)}\right)^{-1} \mu_{1}^{(1)}, \quad x=\overline{1, J}, \\
& \mu_{x}^{(2)}=\lambda_{1}^{(1)}\left(1-\prod_{i=1}^{j} \lambda_{i}^{(2)}\right)\left(\prod_{i=1}^{x} \lambda_{i}^{(2)}\right)^{-1} \mu_{1}^{(1)} \text {, } \\
& x=\overline{\jmath+1, n} \text {. }
\end{aligned}
$$

Then the fuzzy membership function $M^{(1)}$ of the state of the automaton of the subset $L_{1}^{(n)}$, in which the automaton $D_{2 n, 2}^{(j)}$ performs the first action,

$$
\begin{gathered}
M^{(1)}=1-\prod_{x=1}^{n}\left(1-\mu_{x}^{(1)}\right)= \\
=1-\prod_{x=1}^{j}\left(1-\mu_{x}^{(1)}\right) \prod_{x=j+1}^{n}\left(1-\mu_{x}^{(1)}\right)= \\
=1-\prod_{x=1}^{j}\left\{1-\lambda_{1}^{(1)}\left(\prod_{i=1}^{x} \lambda_{i}^{(1)}\right)^{-1} \mu_{1}^{(1)}\right\} \times \\
\times \prod_{x=j+1}^{n}\left\{1-\lambda_{1}^{(1)}\left(1-\prod_{i=1}^{j} \lambda_{i}^{(1)}\right) \times\right. \\
\left.\times\left(\prod_{i=1}^{x} \lambda_{i}^{(1)}\right)^{-1} \mu_{1}^{(1)}\right\} .
\end{gathered}
$$

Similarly, for the second action of the automaton, we obtain:

$$
\begin{gathered}
M^{(2)}=1-\prod_{x=1}^{n}\left(1-\mu_{x}^{(2)}\right)= \\
=1-\prod_{x=1}^{j}\left(1-\mu_{x}^{(2)}\right) \prod_{x=j+1}^{n}\left(1-\mu_{x}^{(2)}\right)=
\end{gathered}
$$

$$
\begin{gathered}
=1-\prod_{x=1}^{j}\left\{1-\lambda_{1}^{(1)}\left(\prod_{i=1}^{x} \lambda_{i}^{(2)}\right)^{-1} \mu_{1}^{(1)}\right\} \times \\
\times \prod_{x=j+1}^{n}\left\{1-\lambda_{1}^{(1)}\left(1-\prod_{i=1}^{j} \lambda_{i}^{(2)}\right) \times\right. \\
\left.\times\left(\prod_{i=1}^{x} \lambda_{i}^{(2)}\right)^{-1} \mu_{1}^{(1)}\right\} .
\end{gathered}
$$

It follows from (16), (17) that

$$
M^{(1)}>M^{(2)} \text {, }
$$

if

$$
\prod_{i=1}^{n} \lambda_{i}^{(1)}<\prod_{i=1}^{n} \lambda_{i}^{(2)} .
$$

To fulfill this inequality, one can also consider various possible cases of comparing the values $\lambda_{x}^{(\alpha)}$ and $\lambda_{x}^{(\beta)}, x=\overline{1, n}, \quad \alpha, \beta=1,2, \alpha \neq \beta$. However, it is certainly fulfilled if condition (10) is satisfied.

\section{Conclusion}

Let us formulate the results of the study of the behavior of automata in a more general form.

Thus, in a non-stationary fuzzy environment, which encourages or punishes the finite automata $L_{2 n, 2}^{(j)}$ and $D_{2 n, 2}^{(j)}$ with some fixed membership functions, which in turn depend on the states of the automata, is observed the fact of learning these automata and they often perform the action $f_{1}$ or $f_{2}$, for which

$$
\begin{gathered}
\max _{x} \lambda_{x}^{(\alpha)}<\min _{x} \lambda_{x}^{(\beta)}, \\
x=\frac{1, n}{}, \quad \alpha, \beta=1,2, \quad \alpha \neq \beta .
\end{gathered}
$$

Moreover, if the memory depth of the automaton $L_{2 n, 2}^{(j)} \quad n \rightarrow \infty$, then the automaton $L_{2 n, 2}^{(j)}$ in a nonstationary fuzzy environment is asymptotically optimal if

$$
\begin{gathered}
\max _{x} \lambda_{x}^{(\alpha)}<1 / 2<\min _{x} \lambda_{x}^{(\beta)}, \\
\text { If } \quad x=\frac{1, n}{1, n} \quad \alpha, \beta=1,2, \quad \alpha \neq \beta . \\
\lambda_{x}^{(\alpha)} \leq 1 / 2 \quad \text { or } \lambda_{x}^{(\alpha)} \geq 1 / 2, \quad \alpha=1,2,
\end{gathered}
$$

then the asymptotically optimal behavior of the automaton $L_{2 n, 2}^{(j)}$ is impossible.

The finite automaton $D_{2 n, 2}^{(j)}$ as $n \rightarrow \infty$ is asymptotically optimal or anti-optimal depending on the initial state of the automaton. Indeed, being in any of the possible states of the subset $L_{\alpha}^{(n)}$ or $L_{\beta}^{(n)}$, $\alpha, \beta=1,2, \quad \alpha \neq \beta$, then at the first punishment the 
can go into the deepest state of the same subset and will forever remain there.

Note that, if $j=1$ and $\lambda_{x}^{(\alpha)}=\lambda_{1}^{(\alpha)}, \forall x=$ $\overline{1, n}, \quad \alpha=1,2$, then the non-stationary fuzzy environment becomes is a stationary fuzzy environment and the results obtained coincide with the results for automata M.L. Tsetlin's $L_{2 n, 2}^{(1)}$ and V.I. Krinsky's $D_{2 n, 2}^{(1)}$ of [8] and [9].

The results obtained can be easily extended to the case of any number actions of automata $L_{2 n, 2}^{(j)}$ and $D_{2 n, 2}^{(j)}$.

We noted above that the theory of probability has serious experimental support in statistics, in contrast to the theory of fuzzy sets. Therefore, the results obtained in the present article can be considered as another example for statistics of fuzzy systems.

\section{References}

[1] Neumann, J., The theory of self-replicating automata. M., Science, 1971.

[2] Tsetlin, M.L., On the behavior of finite automata in random environments. Automation and telemechanics, N10, 1961.

[3] Robbins, H., A Sequential DecisionProblem with a Finite Memory. Proc. Of Nat. Acad. Of Science of USA, 42, 3, 1956.
[4] Krinsky, V.I., Asymptotically optimal automaton with exponential rate of convergence. Biophysics 9, issue 4, 1964. pp. 67-71.

[5] Korolyuk, V.S., Pletnev, A.I., Eidelman, S.D., Automata. Walks. Games. Successes of Mathematical Sciences. T. 43, issue 1 (259), 1988, pp. 87-122.

[6] Zadeh L., Fuzzy sets. Information and control, N8, 1965, pp. 338-348.

[7] Stefanuk, V.L., Generalized Markov chains. Artificial Intelligence and Decision Making, 2011, N4, pp. 95-99.

[8] Stefanuk,V.L, Behavior of The Finite Automaton in a Fuzzy Environment: Theory and Applications. Artificial Intelligence and Decision Making, 2014, N3, pp. 54-61.

[9] Khvedelidze, T., Aslanishvili, I., On the Behavior of One Finite Automaton in a Fuzzy Environment. Published 2021-03-11.

Publisher EAI. http://dx.doi.org/10.4108/eai.27-22020.2303236. Copyright (C) 2020-2021 EAI.

[10].Tsertsvadze, G. N., Stochastic automaton with hysteresis tactics. Proceedings of TSU, 1970, v. 135, p. 57-61.

[11] Stefanuk, V.L., Do you trust the evidence?

All-Union Conference on Artificial Intelligence. T. 1. - M.: USSR Academy of Sciences, 1988. - pp. 406- 410.

[12]Varshavsky,V.I., Collective behavior of automata. M, Science, 1973.

\section{Creative Commons Attribution License 4.0 (Attribution 4.0 International, CC BY 4.0)}

This article is published under the terms of the Creative Commons Attribution License 4.0 https://creativecommons.org/licenses/by/4.0/deed.en US 